\title{
SOBREVIDA DE LOS PACIENTES CON CARCINOMA RENAL AVANZADO TRATADOS CON INTERFERÓN
}

\author{
Yolanda Ares Valdés.
}

Servicio de Urología. Hospital Universitario Manuel Fajardo. Ciudad Habana. Cuba.

Resumen.- OBJETIVO: Determinar la sobrevida de los pacientes con carcinoma renal metastásico tratados con Interferón.

MÉTODOS: Estudio retrospectivo longitudinal de 18 pacientes atendidos en el Hospital Universitario Manuel Fajardo entre julio del 2002 a septiembre del 2007, con diagnóstico de carcinoma renal metastásico tratados con cirugía radical e Interferón Alfa 2 b recombínante adyuvante a la cirugía o como monoterapia.

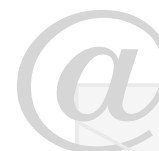

CORRESPONDENCIA

Yolanda Ares Valdés

Indio 113 / Gloria y Corrales

Habana Vieja. Ciudad Habana. (Cuba).

ares@infomed.sld.cu

Trabajo recibido: 25 de febrero 2008
RESULTADOS: El 74\%, 14/18 de los pacientes fueron masculinos, el 26\%, 5/18 femeninos. Al 72\%, 13/18 se les realizó cirugía radical más Interferón y el $28 \%$, $5 / 18$ se le administró el Interferón como monterapía. Se observó respuesta completa en el 22\%, 4/18 y progresión en el 78\%, 14/18. La sobrevida fue del 50\%, $33 \%, 28 \%$ y $22 \%$ a 1, 2, 4 y 5 años respectivamente.

CONCLUSIONES: No se encontró respuesta significativa. No hubo regresión de la carga tumoral en ningún caso con el uso del Interferón. La sobrevida fue mejor en los casos que se les realizó nefrectomía radical más Interferón que en los casos tratados con Interferón como monoterapia.

Palabras clave: Carcinoma Renal. Metastásis. Interferón. Inmunoterapia.

Summary.- OBJECTIVES: To determine survival of patients with metastatic renal carcinoma treated with interferon.

METHODS: Retrospective longitudinal study with 18 patients with the diagnosis of metastatic renal carcinoma treated in the University Hospital Manuel Fajardo between July 2002 and September 2007 with radical surgery and alpha $2 b$ recombinant interferon, either adjuvant or as monotherapy.

RESULTS: $74 \%$ (14/18) of the patients were males, $26 \%(5 / 18)$ females. $72 \%(13 / 18)$ underwent radical surgery plus interferon, and $28 \%(5 / 18)$ received interferon monotherapy.

Complete response was observed in 22\% (4/18); $78 \%$ (14/18) had progression. One, 2, 4 and 5-year survivals were $50 \%, 33 \%, 28 \%$, and $22 \%$, respectively. 
CONCLUSIONS: No significant response was found. There was not tumor regression in any case using interferon. Survival was better in cases undergoing radical nephrectomy plus interferon than cases treated with interferon monotherapy.

Keywords: Renal carcinoma. Metastasis. Interferon. Immunotherapy.

\section{INTRODUCCIÓN}

El cáncer de células renales representa el 3\% de los tumores malignos del adulto (1). La incidencia de la enfermedad metastásica ha permanecido casi inalterada a pesar de los avances en los estudios diagnósticos entre el 30-40\% (2). Los pacientes con Cáncer de Células Renales Metastásico (CCRM) no tratados presentan una sobrevida muy pobre, no mayor de 12 meses; menos del 10\% de los pacientes con esta enfermedad sobreviven a 5 años $(1,2)$.

Algunos estudios han documentado regresión de las metástasis después de la remoción del tumor primario, aunque esto sucede con escasa frecuencia (2). La sobrevida libre de enfermedad aumenta con la resección del tumor primario y una proporción de pacientes permanecen libres de la enfermedad. La nefrectomía paliativa puede ser considerada para aliviar el dolor, la hematuria y el malestar o la hipertensión (3). El CCR es refractario a muchos agentes quimioterapéuticos, en cambio estudios basados en las terapias biológicas han demostrado remisión durable del cáncer renal (4). El uso del Interferón (IFN) alfa presenta una tasa de respuesta (regresión completa o parcial) del $15 \%$, en el caso del CCRM, la respuesta durable es rara (3). El mecanismo preciso de la actividad del IFN no ha sido bien esclarecido y el de la actividad antineoplásica es desconocido. La absorción del IFN alfa $2 b$ recombinante $(r)$ es alta (más del $80 \%$ ) cuando se administra subcutáneo o intramuscular (5). El producto del cual disponemos en este hospital es el IFN alfa $2 b$ r producido en el país.

A pesar de la extensa evolución de diferentes tratamientos en la literatura para el CCRM, este permanece altamente resistente a la terapia sistémica unos pocos pacientes presentan respuesta parcial o completa al IFN pero la mayoría no responden y hay pocos enfermos que sobreviven largo plazo, todo esto nos motivo a realizar este estudio con el objetivo de determinar la supervivencia de los casos de CCRM tratados con cirugía e Interferón.

\section{OBJETIVOS}

\section{General}

Determinar la supervivencia de los pacientes con cáncer de células renales metastásico.

\section{Específicos}

La supervivencia se evaluó en cuanto a:

- Estadio de la enfermedad.

- Tipo de tratamiento

- Nefrectomía radical más inmunoterapia.

- Inmunoterapia aislada.

- Respuesta al tratamiento.

\section{MATERIAL Y MÉTODOS}

\section{Diseño del estudio}

Retrospectivo longitudinal. De julio del 2002 a septiembre del 2007 se trataron 42 pacientes con diagnóstico de CCR en el Hospital Universitario Manuel Fajardo, de ellos 18 casos el $43 \%$ se presentaron como CCRM (localmente avanzado, tumor primario irresecable o metastásico).

\section{Criterios de inclusión}

Clasificación del TNM, sometidos a cirugía radical por vía anterior o no y tratamiento adyuvante con IFN alfa 2 br o como monoterapia, de ambos sexos, edad mayor o igual a 18 años y firma del consentimiento informado.

\section{Criterios de exclusión}

Pacientes con metástasis del sistema nervioso central, sometidos a terapia anti-cáncer, historia de enfermedad maligna en los pasados 5 años $u$ otras enfermedades crónicas, inmunocompromisos que incluye (HIV, hepatitis $B \circ C)$, cirugía previa y/o radioterapia, angina inestable 0 infarto del miocárdico agudo 6 meses antes, uso de anti-arrítmicos, embarazo y lactancia. Se recopilaron 18 expedientes clínicos que cumplían los requisitos del estudio.

\section{Conducción del estudio}

Nefrectomía radical a los tumores resecables y administración de IFN alfa 2 br adyuvante a la cirugía o como monoterapia a los tumores irresecables por vía subcutánea (SC) a una dosis de 20 Millones de Unidades (MU) semanal siete días después del diagnóstico (histológico o clínico) durante 12 semanas que se evaluó una respuesta. Se les realizó chequeo humoral, examen físico y estudios imagenológicos. El tratamiento se continuó por 12 semanas más para obtener una respuesta o la hasta que la enfermedad progreso. 


\section{Criterios de respuesta}

Los criterios internacionales propuestos por el comité de evaluación de la respuesta en tumores sólidos Recist (Journal of Nacional Institute 2000; 92 (3): 205-16) en los que puede realizarse medición de la lesión. Respuesta completa (RC): Desaparición de todas las lesiones dianas. Respuesta parcial: (RP): Reducción de al menos el $30 \%$ de la suma de los diámetros mayores de las lesiones diana, tomando como referencia la suma de diámetros mayores de base. Enfermedad progresiva (EP): Incremento de al menos el $20 \%$ en la suma de los diámetros mayores de las lesiones diana, tomando como referencia la suma más pequeña de diámetros mayores registrada desde el inicio del tratamiento a la aparición de una o más nuevas lesiones. Enfermedad estable: (EE): Reducción no suficiente para calificar como respuesta parcial o incremento insuficiente para calificar como enfermedad progresiva, tomando como referencia la suma más pequeña de diámetros mayores desde el inicio del tratamiento.

Se analizaron las variables siguientes: Sobre-vida a los 1, 2, 4 y 5 años. Variables de control: Edad, sexo, estadio de la enfermedad de la clasificación TNM, nefrectomía radical por vía anterior, inmunoterapia adyuvante con Interferón alfa o como monoterapia. Los datos fueron ingresados en un programa de computación y el análisis estadístico se realizó mediante el Test de Chi cuadrado.

\section{RESULTADOS}

De los 18 casos estudiados, 14/18, el $78 \%$ fueron masculinos y $4 / 18$, el $22 \%$ femeninos, la

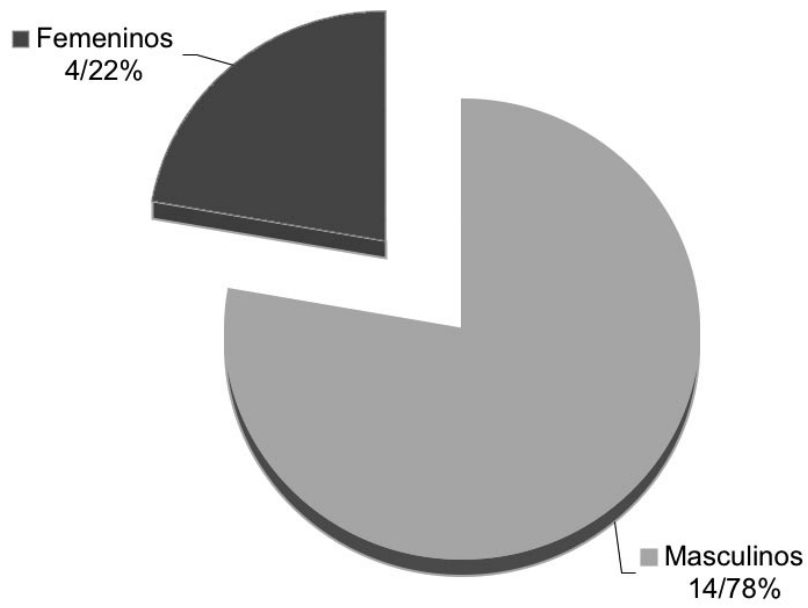

FIGURA 1. Sexo. edad de mayor incidencia fue entre la quinta y séptima décadas con un pico en la sexta $5 / 18$, el $28 \%$ (Figuras 1 y 2).

El $72 \%, 13 / 18$ fueron tratados con cirugía radical más IFN alfa 2 br y el $28 \%, 5 / 18$ con IFN como monoterapia (Figura 3). La respuesta fue completa solamente $4 / 18$, el $22 \%$ y la enfermedad progresó en 14/18, el $78 \%$ de los pacientes a los pocos meses de tratamiento y en los pacientes no quirúrgicos la supervivencia fue muy pobre (Figuras 4 y 5). La sobrevida de los pacientes fue al 1, 2, 4 y 5 años del $56,33,28$ y $22 \%$ respectivamente (Figura 6).

Ningún paciente abandonó la terapia por complicaciones propias del medicamento, todos siguieron su administración hasta su fallecimiento.

\section{DISCUSIÓN}

El carcinoma de células renales se considera la neoplasia más letal de los cánceres urológicos, siendo el responsable del $2 \%$ de los muertos por cáncer renal. En esta serie el $43 \%$ de los casos al momento del diagnóstico se presentaron como CCRM compatible con los estudio revisados (1).

Los grupos de tratamiento no son uniformes y por el contrario los pacientes sometidos a cirugía radical presentaron un mejor estado de salud, en comparación con los casos sometidos a IFN alfa como monoterapia, lo cual explica que el grupo quirúrgico presentó una mejor sobrevida que el no quirúrgico.

El carcinoma renal sigue siendo un grave problema cuyo tratamiento en ciertas etapas, sobre

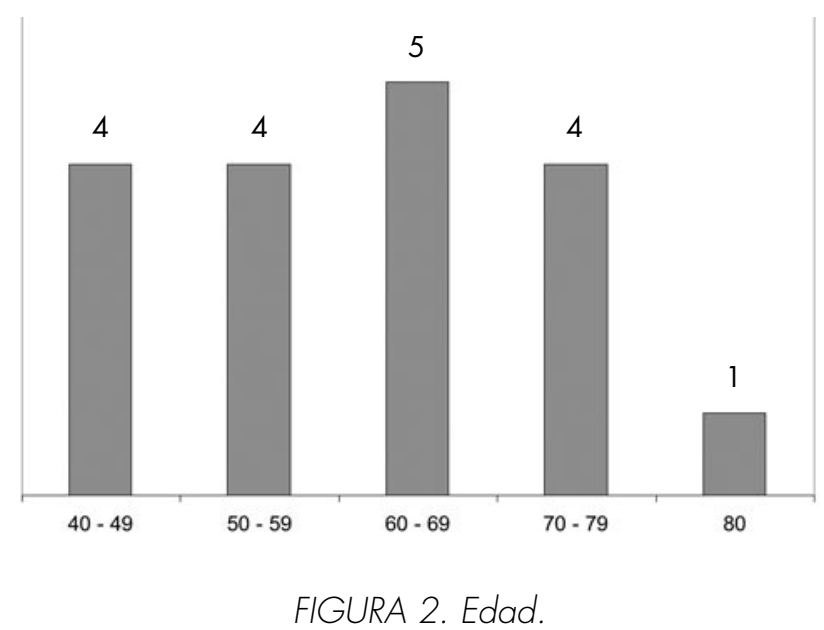




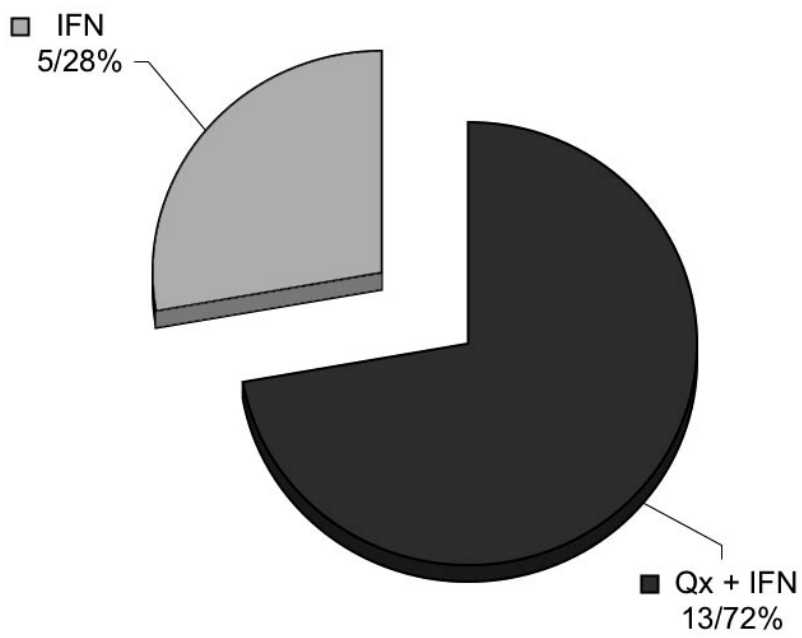

FIGURA 3. Tratamientos.

todo en la enfermedad avanzada se encuentra aun en discusión, la nefrectomía puede ser justificada en los pacientes con CCRM cuando la intensión es mejorar la calidad de vida y mejorar los síntomas que el tumor puede ocasionar, la resección quirúrgica se puede realizar cuando existen metástasis solitarias en este caso la sobrevida puede alcanzar el $30 \%$ a los 5 años (8); en nuestro estudio no se encontraron pacientes que fueran tratados con una resección de una lesión tumoral única.

Flanigan y colaboradores (9) demostraron que el IFN alfa prolonga la sobrevida en pacientes con CCRM cuando se combina con nefrectomía radical. Dos estudios similares; EORTC demostraron que la sobrevida media (nefrectomía más IFN comparado con IFN solo fue de 17 meses Vs 7 meses $(9,10)$. Los

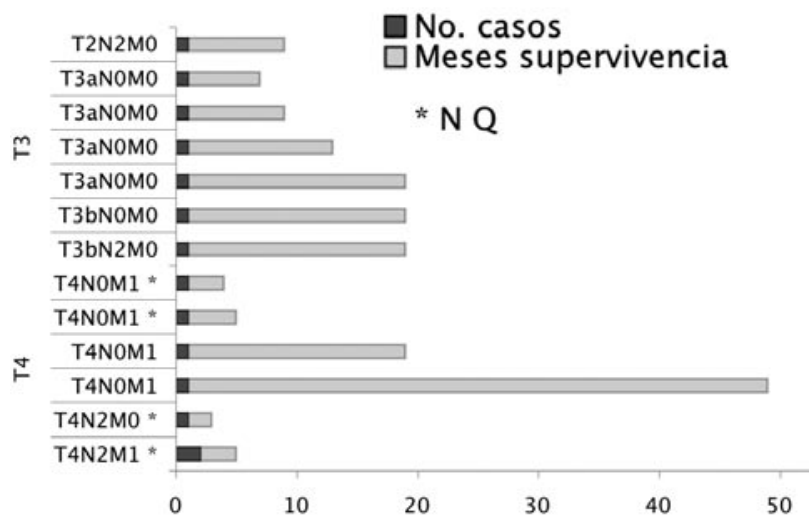

FIGURA 5. Progresión del tumor en pacientes no respondedores.

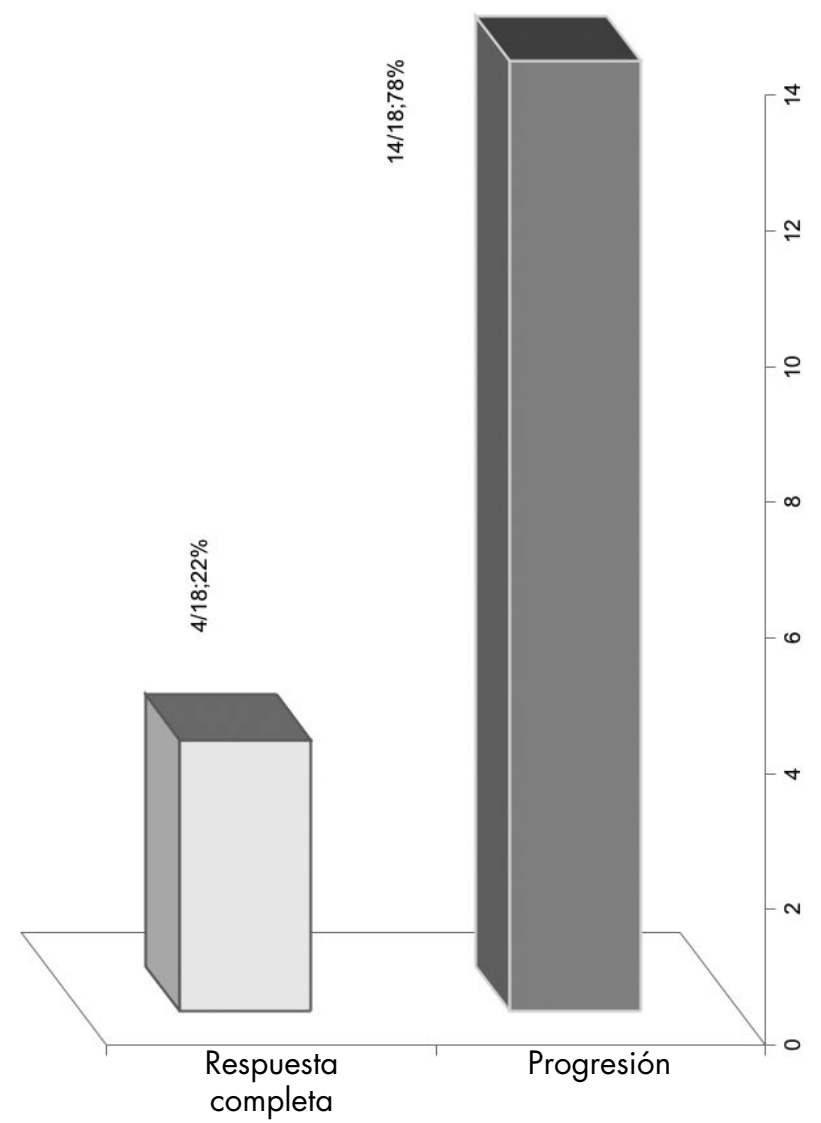

FIGURA 4. Respuesta al tratamiento.

resultados no pueden ser extrapolados, puesto que en los estudios revisados los grupos fueron homogéneos; en nuestro estudio los pacientes con CCRM con mal pronóstico se inicio la inmunoterapia en el momento del diagnóstico y en los pacientes con mejor pronóstico se les realizó cirugía radical e IFN adyuvante, por lo que ya había un sesgo y posibilitaba una sobrevida mayor al grupo de tratamiento quirúrgico.

El CCRM con tratamiento presenta una sobrevida al primer año de menos del $50 \%$, a 5 años del $5-30 \%$ y a los 10 años de 0-5\%. En ningún estudio se ha demostrado que la sobrevida mejore en forma significativa y que los casos que mejor respuesta presentan son aquellos en que el estado de salud es bueno y no presentan múltiples metástasis.

En ningún caso se demostró regresión o disminución de la carga tumoral, no se encontró respuesta significativa. El IFN demostró un bajo grado 


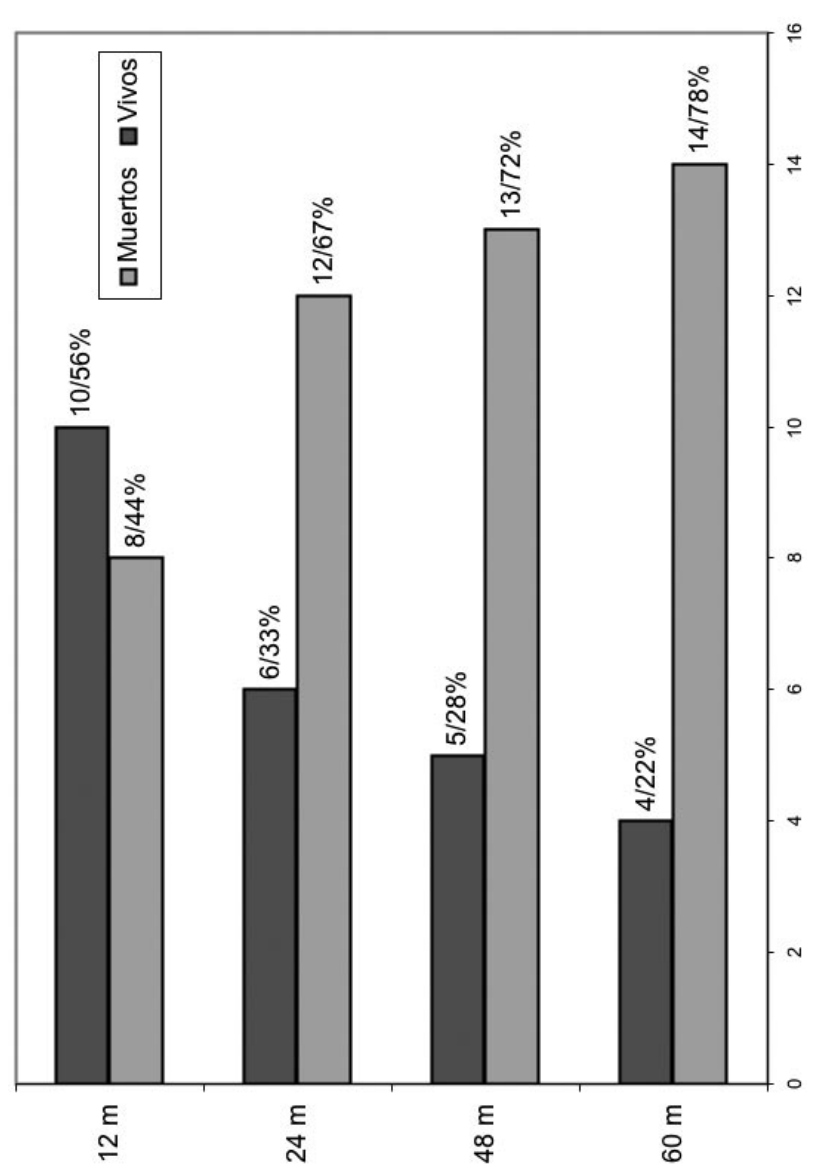

FIGURA 6. Supervivencia.

de efectos antitumoral contra el CCRM, esto se atribuye a que la terapia fue administrada a los pacientes en el estadio final de la enfermedad.

\section{CONCLUSIONES}

1. La sobrevida es mejor en los casos con CCRM tratados con cirugía radical e inmunoterapia adyuvante que en lo manejados con IFN como monoterapia.

2. La inmunoterapia por si sola no ofrece mayor utilidad en la sobrevida, sin que el estado de salud previo influya en los resultados finales.

3. No se observó regresión de la carga tumoral en ningún caso con el uso del IFN.

\section{BIBLIOGRAFÍA y LECTURAS RECOMENDADAS ( ${ }^{*}$ lectura de interés $y^{* *}$ lectura fundamental)}

1. Negrier S, Caty A, Lesimple T, et al. Treatment of patients with metastatic renal carcinoma with combination of subctaneaus interleukin 2 and Interferon alfa with or without fluoraucil. J Clin Oncol 2000;18:4009-15

2. Griffiths T, Mellon J, Evolving immunotherapeutic strategies in bladder and renal cancer. Postgrad Med J 2004;80:320-27

**3. Bukowski RM. Immunotherapy in renal cell carcinoma. Oncology 1999;13:801-10

*4. Negrier S, Escudier B, Lasset C, et al. Recombinant human Interleukin-2 recombinant human Interferon Alfa 2-a or both in metastatic renal cell carcinoma. N Engl J Med 1998; 338.1272-78

*5. Krejci K, Markiewicz M, Kwon E. Immunotherapy for urological malignancies. J Urol 2004;171:870-76

**6. García TM, Sánchez SM, Beltrán AJR. Análisis de supervivencia de los carcinomas de células renales órgano-confinados. Valor pronóstico de la clasificación TNM 1997. Actas Urol Esp 2006;30(7):650-60

**7. Ares VY, Campo AR. Factores pronósticos de la supervivencia en los pacientes intervenidos por cáncer de células renales. Arch Cir Gen Dig, Disponible en: http://www.cirugest.com/revista/2007/07/2007-05-07.htm, 2007

8. Motzer R, Bander N, Nanus D. Medical progress: Renal - cell carcinoma. N Engl J Med 1996;335(12):865-75

**9. Flanigan R, Salmon S, Blumensteint, et al. Nephrectomy followed by interferon A $2 b$ compared with interferon alfa $2 \mathrm{~b}$ alone for metastatic renal cell cancer. N Engle J Med 2001;345:1655-59 